\title{
Kharkiv study of near-Earth asteroids
}

\author{
Yu. N. Krugly ${ }^{1}, 2$, N. M. Gaftonyuk ${ }^{3}$, I. N. Belskaya ${ }^{1}$, V. G. Chiorny ${ }^{1}$, \\ V. G. Shevchenko ${ }^{1}$, F. P. Velichko ${ }^{1}$, D. F. Lupishko ${ }^{1}$, \\ A. A. Konovalenko ${ }^{4}$, I. S. Falkovich ${ }^{4}$ and I. E. Molotov ${ }^{5}$ \\ ${ }^{1}$ Institute of Astronomy of Kharkiv National University, Sumska str. 35, Kharkiv 61022, \\ Ukraine, email: krugly@astron.kharkov.ua \\ ${ }^{2}$ Main Astronomical Observatory, NASU, Zabolotny str. 27, Kyiv 03680, Ukraine \\ ${ }^{3}$ Crimean Astrophysical Observatory, Simeiz 98680, Ukraine \\ ${ }^{4}$ Institute of Radio Astronomy, NASU, Chervonopraporna str. 4, Kharkiv 61002, Ukraine \\ ${ }^{5}$ Keldysh Institute of Applied Mathematics, RAS, Miusskaya sq. 4, Moscow 125047, Russia
}

\begin{abstract}
The regular CCD observations of near-Earth asteroids (NEAs) in the Institute of Astronomy of Kharkiv National University were initiated in 1995 within the framework of asteroid hazard problem in collaboration with the DLR, Institute of Planetary Research (Berlin). The main aim of the study is a determination of rotation periods and shapes of NEAs as well as astrometry of newly discovered objects. We also carry out the absolute photometry of NEAs in $B V R I$ bands in order to put constraints on surface properties and to estimate their diameters. The observations are carried out with 0.7-m telescope of the Institute of Astronomy (Kharkiv) and with 1-m telescope of the Crimean Astrophysical Observatory (Simeiz) in the standard Johnson-Cousins photometric system. Some observations were made as an optical support of radar observation of NEAs. We present the results of photometric observations of 21 NEAs obtained in 2004-2006 which include asteroid rotation properties, diameters and shapes.
\end{abstract}

Keywords. Photometry; asteroid, lightcurves; asteroid, rotation

\section{Introduction}

The regular CCD observations of NEAs were initiated in Institute of Astronomy of Kharkiv National University in 1995 within the framework of collaboration with the DLR, Institute of Planetary Research (Berlin). The main aim of the study is determination of rotation properties and estimation of shapes and sizes of NEAs as well as carrying out astrometry of newly discovered ones.

A possibility of ground-based photometric observations of NEAs during their close approaching the Earth occurs usually once per tens years. It makes difficult an accumulation of information about these bodies. Therefore, the observations of newly discovered NEAs were the most important part of the reported project. We tried to observe NEAs in a wide range of aspect and phase angles to constrain their rotation and shapes. The absolute photometry together with the measurements of color indices was used to estimate diameters and surface properties of the bodies.

We present the results of the CCD observations of 21 NEAs obtained in 2004-2006.

\section{Observations}

The observations were carried out in the standard Johnson-Cousins UBVRI photometric system (mainly in the $\mathrm{R}$ band as the most effective for CCD), using the 0.7-m telescope at the Chuguevskaya Station (70-km to the south-east of Kharkiv) and the 1-m telescope at the Crimean Astrophysical Observatory (Simeiz). Several CCD cameras (SBIG ST-6, 
Apogee Alta U42, FLI IMG1024S and CCD47-10) were used in Newtonian focus of the 70-cm telescope (f/4) and in the Cassegrain focus of the 1-m telescope equipped with a focal reducer (f/5). The coordinated observations from the two sites (Kharkiv and Simeiz), made with the same routine of observations and reduction, promote the obtained results which are less dependent from weather conditions.

All the asteroids were observed during the maximum interval of their night visibility, usually during two-three consecutive nights, to constrain the rotation period. To achieve a good signal-to-noise ratio, the integration time for each image was chosen to range from $10 \mathrm{~s}$ to $360 \mathrm{~s}$, depending on the asteroid magnitude, magnitudes of the comparison stars in the field of view, and the moving rate of the asteroid. Differential photometry of an asteroid in the CCD frame provides an uncertainty of measurements of the order of 0.01-0.03 mag (rms). To compensate, at least partially, the asteroid motion during the exposure, and thus to increase efficiency of the observations, a correction of the telescope tracking was applied. Reduction of the observations and the aperture photometry routine were made in a standard way with the AstPhot software developed by S. Mottola (Mottola et al. 1995). In the case of the asteroid fast motion the method of overlapping CCD fields was used (Krugly 2004). Special attention was paid to absolute magnitude calibration which was fulfilled during nights with the best photometric conditions. The method of observations and data reduction was described in more details in Krugly et al. (2002).

\section{Results}

Since 2004 the observations of 21 NEAs were carried out. Their results are presented in Table 1, which contains year of observations, the absolute magnitude, the diameter estimate, the rotation period, and the maximal observed lightcurve amplitude. Figures 1-5 illustrate a variety of rotation periods and amplitudes of the observed sample of near-Earth asteroids.

The most important results of the project can be shortly summarized as following:

- estimations of rotation periods for 13 NEAs. For (8567) $1996 \mathrm{HW}_{1}$, (66251) 1999 $\mathrm{GJ}_{2}, 2005 \mathrm{AB}$, and $2006 \mathrm{BQ}_{6}$ they have been determined for the first time; more accurate period definitions were obtained for (3200) Phaethon, (23187) $2000 \mathrm{PN}_{9}$, and (54509) $2000 \mathrm{PH}_{5}$. The observations of asteroid (54509) $2000 \mathrm{PH}_{5}$ can be also used for investigation of an influence of the Yarkovsky-O'Keefe-Radzievskii-Paddack (YORP) effect (Bottke et al. 2002).

- determinations of the absolute magnitudes and diameters of 15 NEAs.

- measurements of the B-V, V-R, and R-I colors for about a half of the observed asteroids.

- determinations of NEA lightcurve amplitudes which characterize the asteroid shape elongation. Some of NEAs, like (1685) Toro, (11405) $1999 \mathrm{CV}_{3}$, (54509) $2000 \mathrm{PH}_{5}$, and $2006 \mathrm{BQ}_{6}$ ) show amplitudes as large as 1.0-1.65 mag.

- photometric observations of the newly discovered asteroids $2005 \mathrm{AB}, 2005 \mathrm{CV}_{69}$, and $2006 \mathrm{BQ}_{6}$ during their close approaches the Earth.

- observations at different aspects and phase angles in order to constrain shape, pole coordinates, and surface properties of the following NEAs: (1036) Ganymed, (1627) Ivar, (1862) Apollo, (1685) Toro, (1980) Tezcatlipoca, (3103) Eger, (3200) Phaethon, (4179) Toutatis, and (11405) $1999 \mathrm{CV}_{3}$. The asteroids Ganymed and Ivar were observed down to phase angles as small as 1.5 and $3.5 \mathrm{deg}$, respectively.

- photometric and astrometric observations in support of the radar observations of the NEAs (4179) Toutatis, $2000 \mathrm{PH}_{5}$, and $2004 \mathrm{XP}_{14}$ during their close approaching the 
Table 1. Results of NEA photometry

\begin{tabular}{lcccclc}
\hline Asteroid & $\begin{array}{c}\text { Orbit } \\
\text { type }\end{array}$ & $\begin{array}{c}\text { Year of } \\
\text { observ. }\end{array}$ & $\begin{array}{c}H \\
(\mathrm{mag})\end{array}$ & $\begin{array}{c}D \\
(\mathrm{~km})\end{array}$ & $\begin{array}{l}\text { Rotation period } \\
(\mathrm{hrs})\end{array}$ & $\begin{array}{c}\text { Amplitude } \\
(\mathrm{mag})\end{array}$ \\
\hline 1036 Ganymed & Amor & 2006 & 9.58 & 39 & $10.314 \pm 0.004$ & 0.12 \\
1627 Ivar & Amor & 2005 & 13.06 & 8.4 & $4.7956 \pm 0.0002$ & 0.35 \\
1685 Toro & Apollo & 2004 & $(14.23)$ & 3.5 & 10.19 & $>1.0$ \\
1862 Apollo & Apollo & 2005 & $16.1^{*}$ & 1.6 & $3.065 \pm 0.005$ & 0.26 \\
1980 Tezcatlipoca & Amor & 2006 & 13.78 & 6.1 & $7.246 \pm 0.009$ & 0.58 \\
3103 Eger & Apollo & 2006 & $(15.38)$ & 2.5 & 5.706 & 0.7 \\
3200 Phathon & Apollo & 2004 & $14.52^{*}$ & 6.7 & $3.6052 \pm 0.0008$ & 0.17 \\
4179 Toutatis & Apollo & 2004 & 15.38 & 2.8 & $176.4^{1}$ & 0.8 \\
6611 1993 VW ${ }^{B}$ & Apollo & 2005 & 16.61 & 1.0 & $2.556^{2}$ & 0.06 \\
8567 1996 HW1 & Amor & 2005 & $15.04^{*}$ & 3.5 & $8.75 \pm 0.05$ & 0.4 \\
100085 1992 UY4 & Apollo & 2005 & 17.72 & 1.55 & $12.88 \pm 0.06$ & $>0.5$ \\
11405 1999 CV3 & Apollo & 2006 & 15.73 & 2.45 & $6.507 \pm 0.006$ & 1.1 \\
13553 1992 JE & Amor & 2005 & 16.0 & 3.4 & $>30$ & $>0.7$ \\
23187 2000 PN9 & Apollo & 2006 & $(16.1)$ & 2.1 & $2.5325 \pm 0.0004$ & 0.13 \\
54509 2000 PH5 & Apollo & 2004 & 22.50 & 0.1 & $0.202875 \pm 0.00003$ & 1.0 \\
66251 1999 GJ2 & Amor & 2005 & $17.1^{*}$ & 1.1 & $2.462 \pm 0.001$ & 0.10 \\
1999 LF6 & Apollo & 2004 & $(18.2)$ & 0.8 & $>12$ & 0.3 \\
2004 XP14 & Apollo & 2006 & $(19.4)$ & 0.45 & $>24$ & $\sim 0.05$ \\
2005 AB & Amor & 2005 & $(17.5)$ & 2. & $3.346 \pm 0.008$ & 0.11 \\
2005 CV69 & Apollo & 2005 & 18.1 & 1.3 & - & \\
2006 BQ6 & Apollo & 2006 & 19.45 & 0.44 & $4.414 \pm 0.008$ & 1.65 \\
\hline
\end{tabular}

Note: $H$ values in brackets were adopted from the Minor Planet Circulars. Diameters were calculated assuming an albedo 0.15 , if the asteroid albedo and type were unknown.

${ }^{B}$ Supposed to be binary.

* The absolute magnitude was obtained from $\mathrm{R}$-band observations and the $\mathrm{V}-\mathrm{R}=0.43$ was used to transform $H_{R}$ to $H$.

${ }^{1}$ Period of the long-axis precession Hudson \& Ostro (1995).

${ }^{2}$ Pravec (2005, private communication).

Earth. On 2006 July 3 the international radar experiment was arranged to investigate the NEA $2004 \mathrm{XP}_{1} 4$ with participation of radar and antenna facilities in USA, Ukraine, Russia, Italy and China. The object was sounded with RT-70 in Goldstone (USA) at $3.6 \mathrm{~cm}$ and with RT-70 in Evpatoria (Ukraine) at $6 \mathrm{~cm}$ wavelength. The outside of USA the echoes were detected with RT-64 in Kalyazin (Russia) at $6 \mathrm{~cm}$ and with RT-70 in Evpatoria at $3.6 \mathrm{~cm}$ (see Figure 6 which presents the first result of the radar experiment).

- observations of four binary NEAs: (1862) Apollo and 2006 BQ 6 identified as binaries from radar observations; (6611) $1993 \mathrm{VW}$ and $2005 \mathrm{AB}$ found out to be binary in the Photometric Survey for Asynchronous Binary Asteroids Project (Pravec 2005). Additionally, observations of (1862) Apollo helped to determine the YORP effect in the rotation of this asteroid (Kaasalainen et al., 2006, to be submitted).

\section{Conclusions and perspectives}

The regular CCD observations of NEAs in Kharkiv have been launched since 1995 . It was the beginning of follow-up program of NEAs study in Ukraine. The observations were carried out in the Institute of Astronomy of the Kharkiv National University in cooperation with the Crimean Astrophysical Observatory (Simeiz). Over 500 lightcurves of more than 100 NEAs have been obtained. Recently the larger and more sensitive CCD cameras have become available for both observation sites (Kharkiv and Simeiz), which noticeably improve our potential to investigate key properties of the NEA population. 

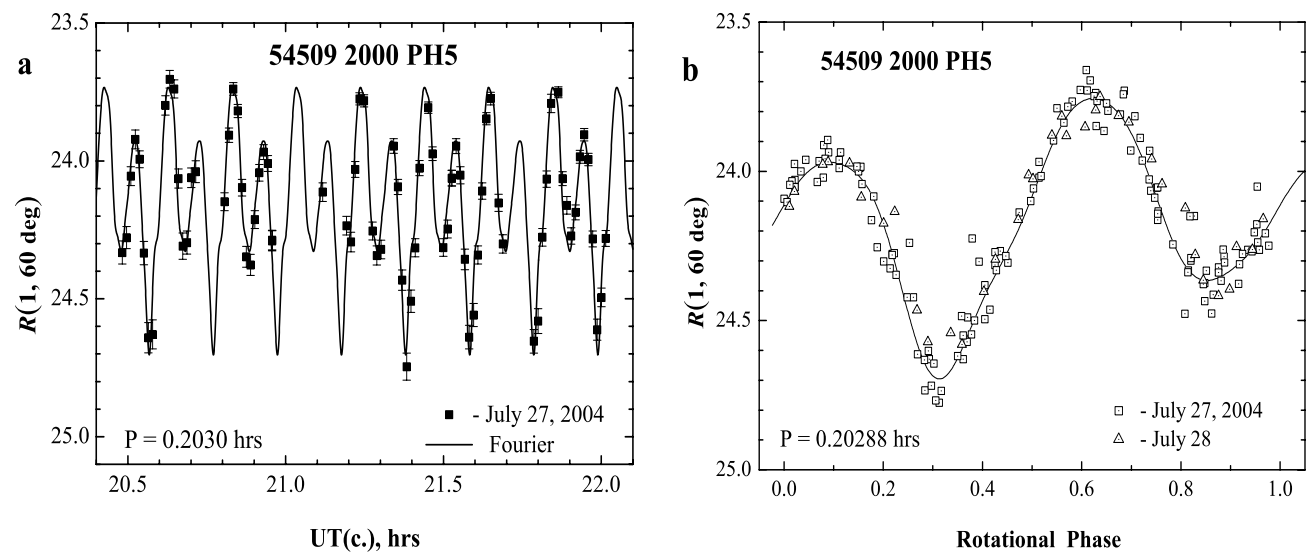

Figure 1. Individual lightcurve of the fast-rotating asteroid (54509) $2000 \mathrm{PH}_{5}$ on July 27, 2004 with the best 6 th order Fourier series fitting (a). The composite lightcurve of the asteroid obtained from two consecutive nights in July 2004 (b).
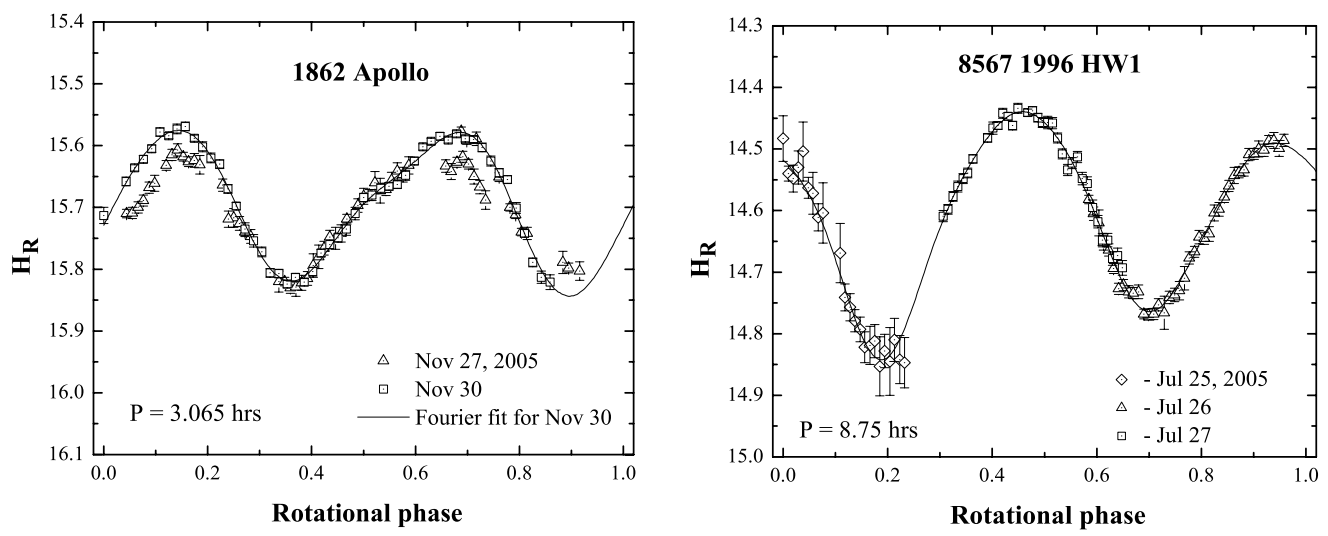

Figure 2. Composite lightcurves of NEAs: (1862) Apollo observed in November 2005 (left); (8567) $1996 \mathrm{HW}_{1}$ observed in July 2005 (right).
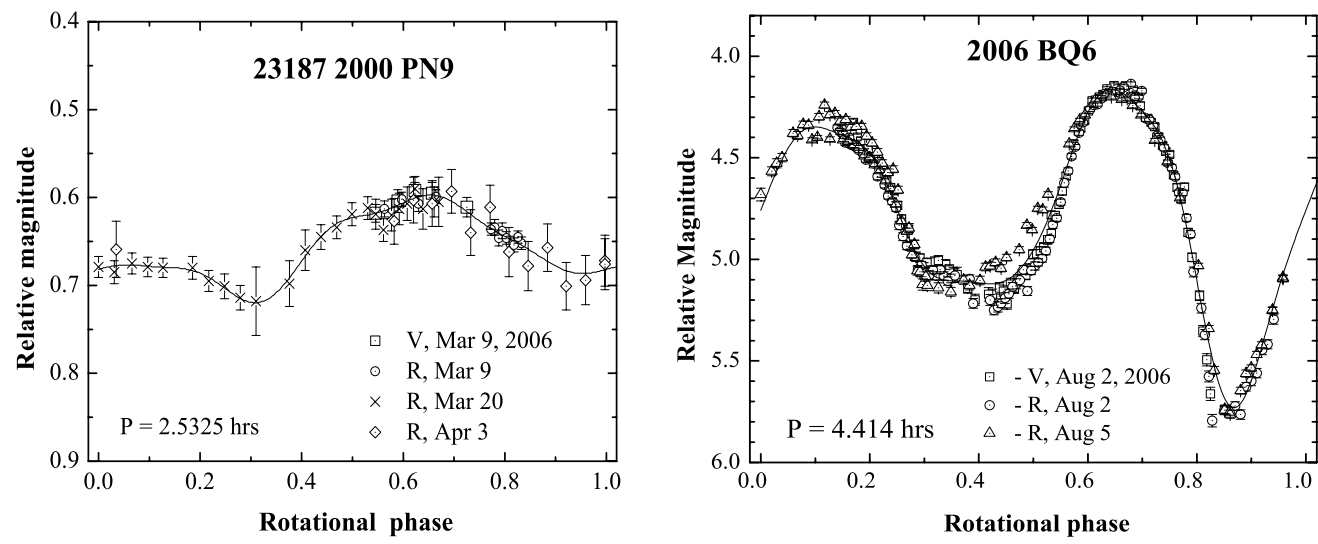

Figure 3. Composite lightcurves of NEAs: (23187) $2000 \mathrm{PN}_{9}$ observed in March-April 2006 (left); $2006 \mathrm{BQ}_{6}$ observed in August 2006 (right). 

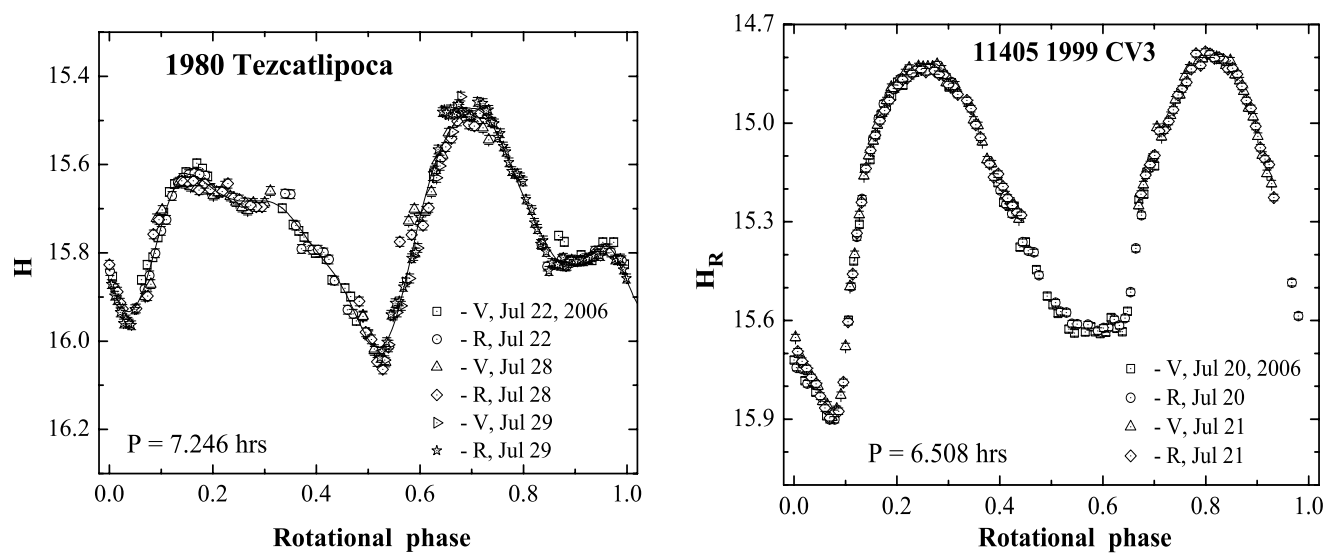

Figure 4. Composite lightcurves of NEAs: (1980) Tezcatlipoca observed in July 2006 (left); (11405) $1999 \mathrm{CV}_{3}$ observed in July 2006 (right).
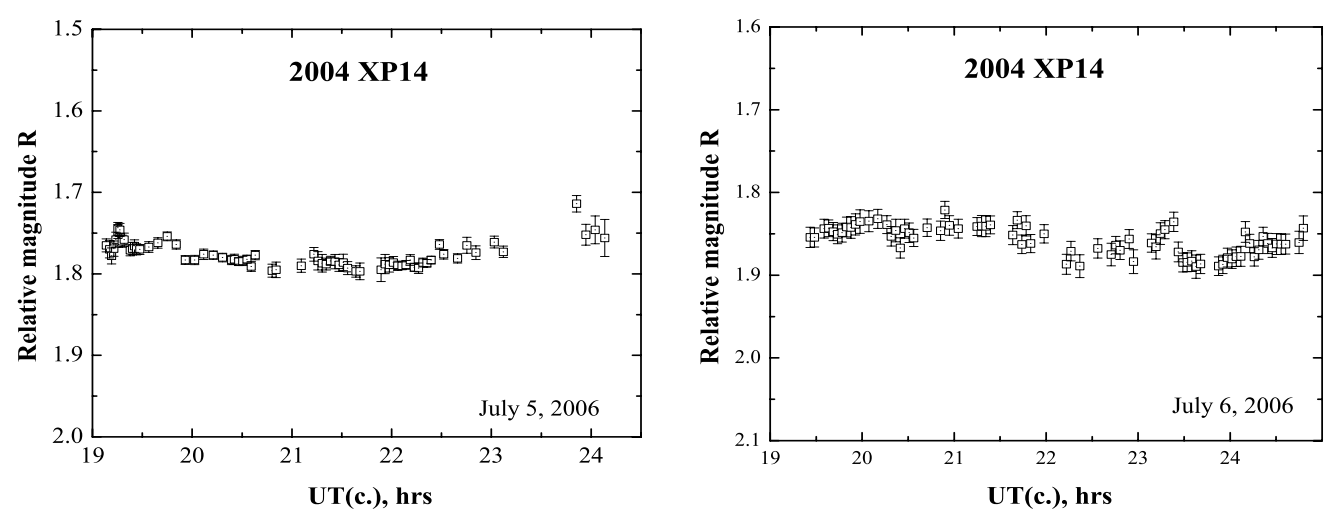

Figure 5. Two individual lightcurves of NEA $2004 \mathrm{XP}_{14}$ observed on July 5 and 6, 2006.

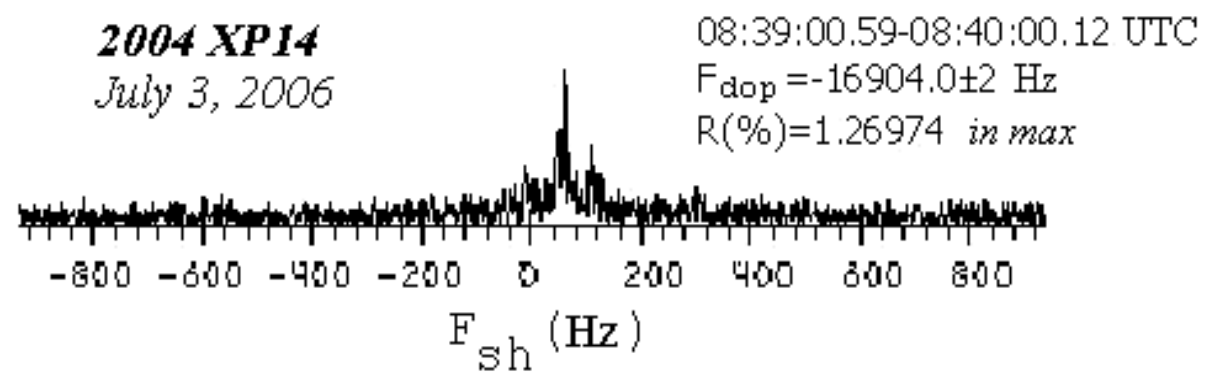

Figure 6. The measurement of Doppler shift of NEA 2004 XP14 echo received with Evpatoria RT-70. The echo-signal is cross-correlated with model of sounding signal of Goldstone RT-70; correlation coefficient $R$ (y axes) is plotted vs. Doppler shift Fsh (x axes).

Last years we have provided an optical support of radar observations of the selected NEAs with RT-70 antenna in Evpatoria (Ukraine) (Konovalenko et al. 2005). Such collaboration seems to be very promising. 


\section{Acknowledgements}

Yu.N.K. and D.F.L. are thankful to IAU for awarding grants to attend the XXVIth General Assembly and, personally, to Oddbjorn Engvold and Monique Orine for their helpful assistance. This work was partially supported by Ministry of Education and Science of Ukraine. Since June 2006 observations in Kharkiv were carried out with CCD camera obtained thanks to INTAS grant Ref. No 03-70-567.

\section{References}

Bottke, W. F., Vokrouhlický, D., Rubincam, D. P. \& Brǒ̌, M. 2002, in: W. F. Bottke, A. Cellino, P. Paolicchi \& R. P. Binzel (eds.), Asteroids III (Tucson: University of Arizona Press), p. 395

Hudson, R. S. \& Ostro, S. J. 1995, Science 270, 84

Konovalenko, A. A., Falkovich, I. S., Lytvynenko, L. M., Nabatov, A. S., Petrenko, A. G., Fedorov, A. P., Kamelin, A. B., Malevinskyi, S. V., Molotov, I. E., Dement'ev, A. F., Lipatov, B. N., Nechaeva, M. B., Tukkari, J., Lu, Sh., Gorshenkov, Yu. N. \& Agapov, V. M. 2005, Radio Physics and Radio Astronomy 10, Special issue, S20

Krugly, Yu. N., Belskaya, I. N., Shevchenko, V. G., Chiorny, V. G., Velichko, F. P., Erikson, A., Mottola, S., Hahn, G., Nathues, A., Neukum, G., Gaftonyuk, N. M. \& Dotto, E. 2002, Icarus 158, 294

Krugly, Yu. N. 2004, Solar System Research 38 (3), 241

Mottola, S., De Angelis, G., Di Martino, M., Ericson, A., Hahn, G. \& Neukum, G. 1995, Icarus 117,62

Pravec P. 2005, in: B. D. Warner, D. Mais, D. A. Kenyon \& J. Foote (eds.), Proceedings for the 24th Annual Conference of the Society for Astronomical Science (Published by Society for Astronomical Sciences, Inc.), p. 61 